\title{
BMJ Open Protocol for a prospective, international cohort study on the Management and Outcomes of Perioperative Care among European Diabetic Patients (MOPED)
}

\author{
Donal J Buggy (D) , ${ }^{1,2,3}$ Rachel Nolan (D) , ${ }^{1}$ Mark Coburn, ${ }^{4}$ Malachy Columb, ${ }^{5}$ \\ Jeroen Hermanides, ${ }^{6}$ Markus W Hollman, ${ }^{6}$ Alexander Zarbock (iD ${ }^{7}$
}

To cite: Buggy DJ, Nolan R, Coburn M, et al. Protocol for a prospective, international cohort study on the Management and Outcomes of Perioperative Care among European Diabetic Patients (MOPED). BMJ Open 2021;11:e044394. doi:10.1136/ bmjopen-2020-044394

- Prepublication history for this paper is available online. To view these files, please visit the journal online (http://dx.doi. org/10.1136/bmjopen-2020044394).

Received 08 September 2020 Accepted 28 July 2021

Check for updates

(C) Author(s) (or their employer(s)) 2021. Re-use permitted under CC BY-NC. No commercial re-use. See rights and permissions. Published by BMJ.

For numbered affiliations see end of article.

Correspondence to

Prof. Donal J Buggy;

donal.buggy@ucd.ie

\section{ABSTRACT}

Introduction Diabetes is common (about 20 million patients in Europe) and patients with diabetes have more surgical interventions than the general population. There are plausible pathophysiological and clinical mechanisms suggesting that patients with diabetes are at an increased risk of postoperative complications. When postoperative complications occur in the general population, they increase major adverse events and subsequently increase 1-year mortality. This is likely to be worse in patients with diabetes. There is variation in practice guidelines in different countries in the perioperative management of patients with diabetes undergoing major surgery and whether this may affect postoperative outcome has not been investigated on a large scale. Neither is it known whether different strata of preoperative glycaemic control affects outcome.

Methods and analysis A prospective, observational, international, multicentre cohort study, recruiting 5000 patients with diabetes undergoing elective or emergency surgery in at least $n=50$ centres. Inclusion criteria are any patient with diabetes undergoing surgery under any substantive anaesthetic technique. Exclusion criteria are not being a confirmed diabetic patient and patients with diabetes undergoing procedures under monitored sedation or local anaesthetic infiltration only. Followup duration is 30 days after surgery. Primary outcome is days at home at 30 days. Secondary outcomes are Comprehensive Complications Index, Quality of Recovery (QoR-15) score on Day 1 postoperatively, 30-day mortality, length of hospital stay and incidence of specific major adverse events (Myocardial Infarction (MI), Myocardial Injury after Non-cardiac Surgery (MINS), Acute Kidney Injury (AKI), Postoperative Pulmonary Complications (PPC), Cerebrovascular Accident (CVA), Pulmonary Embolism (PE), DVT, surgical site infection, postoperative pulmonary infection). Tertiary outcomes include time to resumption of normal diabetes therapy, incidence of diabetic ketoacidosis or hypoglycaemia, incidence and duration of use of intravenous insulin infusion therapy and change in diabetic management at 30 days.

Ethics and dissemination This study will adhere to the principles of the Declaration of Helsinki (amendment 2013) by the World Medical Association and the ICHGood Clinical Practice (GCP) Guidelines E6(R2). Specific national and local regulatory authority requirements

\section{Strengths and limitations of this study}

- This will be the largest prospective, observational study of the perioperative anaesthetic management of patients with diabetes, documenting the influence of perioperative management on 30-day outcomes.

- The primary endpoint is days at home at 30 days, which is a recently validated standardised endpoint for perioperative trials (range 0-30 days, higher number indicating better outcome) that gives a patient-centred outcome reflecting mortality, postoperative complications and return to independent living.

- Secondary outcomes include Comprehensive Complications Index, a scale ranged 0-100, higher number indicating worse outcome, based on the Clavien-Dindo Scale of postoperative complications.

- Broad inclusion criteria include confirmed diabetic patients undergoing any surgery under any substantive anaesthetic technique, which will enhance the external validity of the trial results and render it generalisable on a global scale.

- The power of this study is driven by the target number 5000 patients, which will enable more than 60 variables to be evaluated and up to 11 a priori hypotheses to be tested.

will be followed as applicable. Ethical approval has been granted by the Institutional Review Board of the Mater Misericordiae University Hospital, Dublin, Ireland (Reference: $1 / 378 / 2167)$. As enrolment for this study is ongoing, ethical approval from additional centres is being added continuously. The main results of Management and Outcomes of Perioperative Care among European Diabetic Patients and its substudies will be published in peerreviewed international medical journals and presented at Euroanaesthesia congress and other international and national meetings.

Trial registration number NCT04511312.

\section{INTRODUCTION}

The incidence of diabetes is increasing globally, with an estimated 20 million patients with diabetes in Europe. This is likely to increase, 
adding to societal demands on European health services. ${ }^{1}$ Patients with diabetes are more likely to have surgical interventions than the general population. ${ }^{2}$ There are plausible pathophysiological and clinical mechanisms that patients with diabetes are at an increased risk of postoperative complications. ${ }^{34}$ When postoperative complications occur in the general population, they increase mortality or risk of major adverse cardiovascular events (myocardial infarction, cerebrovascular accident, pulmonary embolism) at 30 days and up to 1 year later. ${ }^{5-7}$ In addition, diabetes is an independent risk factor for surgical site infections. ${ }^{6}$

National bodies in Europe and elsewhere differ in their guidelines on management of patients with diabetes undergoing surgery and small observational studies confirm wide variability in practice and perioperative management between centres. ${ }^{38}$ Given the multiplicity of guidelines and differing recommendations, it is unsurprising that variability of 'real-world' clinical practice regarding perioperative management of oral antihyperglycaemic medications and insulin therapy has been observed in audits such as the National Confidential Enquiry into Patient Outcome and Death. ${ }^{9}$ Whether this variability in practice affects postoperative outcome among patients with diabetes in Europe or elsewhere has not been investigated.

Further, although it is assumed that patients with diabetes are at an increased risk of postoperative complications, ${ }^{5-8}$ this has not been evaluated recently, especially in light of ongoing developments in perioperative care, such as enhanced recovery programmes. ${ }^{7}$ While a quality improvement intervention study has shown that maintaining tight preoperative glycaemic control improves postoperative glycaemic control, ${ }^{10}$ it is not known if this reduces postoperative morbidity overall. Moreover, whether certain anaesthetic techniques may be associated with better or worse outcomes after major non-cardiac surgery is unknown.

Subgroup analysis will provide novel data on how patients with different strata (levels) of preoperative glycaemic control progress in the postoperative period. Poor preoperative glycaemic control is associated with postoperative complications in retrospective studies. ${ }^{1011}$ If this prospective study confirms an association between the level of preoperative glycaemic control and postoperative outcome, then the beginning of personalised perioperative medicine for patients with diabetes might be enabled. For example, it is known from intensive care medicine that patients with better preadmission glycaemic control (haemoglobin A1c (HbAlc)<53 mmol·mol) have worse outcomes if they develop hyperglycaemia, compared with patients whose pre-existing glycaemic control was already poor $(\mathrm{HbA} 1 \mathrm{c}>69 \mathrm{mmol} \cdot \mathrm{mol}){ }^{4}{ }^{41}$

This large, multicentre, international, prospective observational study will address these urgent research questions and will inform better management and outcomes for patients undergoing surgery with this high risk, highly prevalent condition, which is increasing in incidence in the European population.

\section{OBJECTIVES}

To address the following research questions:

1. What is the epidemiology of patients with diabetes undergoing surgery across Europe: Are there major variations in perioperative glycaemic control? Does management practice vary between nations?

2. What is the extent and patient-centred impact of postoperative complications among patients with diabetes up to 30 days after surgery in Europe?

3. To undertake subgroup analysis comparing:

a. Type 1 , type 2 and other patients with diabetes.

b. Patients with different strata (levels) of glycaemic control, that is, HbAlc $<53 \mathrm{mmol} \cdot \mathrm{mol}, \mathrm{HbAlc} 53-69$ $\mathrm{mmol} \cdot \mathrm{mol}$ and $\mathrm{HbA} 1 \mathrm{c}>69 \mathrm{mmol} \cdot \mathrm{mol}$.

c. Patients who received different anaesthetic techniques: Volatile versus total intravenous anaesthesia and regional versus general anaesthesia (GA).

d. Whether patients with diabetes of longer duration versus more recently diagnosed patients with diabetes have higher risk of intraoperative hypotension due to autonomic neuropathy.

\section{METHODS AND ANALYSIS}

Overall study design-Management and Outcomes of Perioperative Care among European Diabetic Patients (MOPED) is a prospective, observational, international, multicentre cohort study, supported by the European Society of Anaesthesiology (ESA).

Setting-Any hospital in Europe (as defined by the $\mathrm{WHO}$ ) is welcome to participate as a study centre. NonEuropean centres may be accepted on request to the steering committee (SC). Centres will be asked to enrol a minimum of 45 patients, in order to nominate one named coinvestigator. The recruitment period will be up to 18 months from the date of the centre's registration with ESA. No more than one quarter (25\%) of a centre's patients can be day cases (ambulatory anaesthesia). Study centre registration will occur online via the dedicated 'Call for Centres form' on the ESA website. The start of recruitment for individual centres should be as soon as possible after centre registration with ESA, provided that there is prior institutional review board (IRB) approval. It is envisaged that at least $n=50$ centres will actively enrol patients. It is hoped that patients from at least 10 nations will be enrolled. Enrolment will continue until the planned sample size $(n=5000)$ has been reached.

National co-ordinating investigators are anaesthesiologists appointed by ESA and the SC to lead the project within individual countries. Their responsibility includes: Identifying participating centres in their country and recruiting local co-ordinators in participating hospitals; ensuring all necessary national or regional regulatory approvals are in place prior to start of patient inclusion and facilitating good communication between ESA headquarters and the participating sites in that nation. Local centre co-ordinators may be anesthesiologists, surgeons or diabetes physician working in perioperative medicine who will ensure all relevant 
regulatory/ethical approvals are in place for their institution and who will supervise enrolment, data collection and adjudicate morbidity events.

\section{Participants}

Inclusion criteria-patients with diabetes (all classes except gestational diabetes) undergoing surgery with a substantive anaesthetic technique will be included. A substantive anaesthetic technique is defined as one requiring any GA or any specific regional anaesthetic technique or a combination. Ambulatory, elective or emergency surgery and patients who receive postoperative care in intensive care or high dependency units will be included. Predefined subgroups of patients with diabetes will be highlighted for later analysis.

Exclusion criteria-Patients who are not diabetic; patients with gestational diabetes and patients undergoing surgery without a substantive anaesthetic technique, that is, surgery under local anaesthetic infiltration or topical anaesthesia alone with or without monitored sedation.

Criteria for withdrawal or discontinuation of participants-Due to the observational nature of the study, the protocol does not define any withdrawal/discontinuation criteria. Patients electing to withdraw from the study may do so at any point. In this case, no further data will be collected. Previously collected, encoded data will be anonymised and analysis may be performed up to the point of data collection. Withdrawing participants will not be replaced, provided that their number does not exceed $5 \%$ of the projected sample size at the end of the planned recruitment period.

Participant information and informed consentWritten, informed consent, using the approved informed consent form, will be sought from each patient prior to inclusion unless an explicit, written exemption by the responsible IRB is provided. A patient information leaflet (PIL) will be provided to patients and must be subject to local IRB review and approval.

\section{Endpoints}

\section{Primary endpoint}

DAH-30 has been validated by a large scale cohort study ${ }^{12}$ as an endpoint which is pragmatic and easily obtained. It is affected by both patient factors (poor function, comorbidities) and surgical technique. DAH-30 is sensitive to surgical risk and impact of postoperative complications in that it accounts for both delayed discharge and readmission $^{13}$ (table 1).

\section{Secondary endpoints}

- Comprehensive Complications Index (CCI) Score, based on Clavien-Dindo Scale. ${ }^{14} 15$

- Quality of Recovery Scale (QoR-15), only taken from patients who are in hospital the day after surgery, that is, day 1 postoperatively. ${ }^{16}$

- 30-day mortality.

- Length of stay in hospital.

\begin{tabular}{|c|c|c|}
\hline Primary & Secondary & Tertiary \\
\hline \multirow[t]{6}{*}{$\begin{array}{l}\text { Days at } \\
\text { home at } 30 \\
\text { days }\end{array}$} & $\begin{array}{l}\text { Comprehensive } \\
\text { Complications } \\
\text { Index }\end{array}$ & $\begin{array}{l}\text { Time to resumption of } \\
\text { normal diabetes therapy }\end{array}$ \\
\hline & $\begin{array}{l}\text { Quality of } \\
\text { Recovery Scale } \\
\text { (QoR-15) }\end{array}$ & $\begin{array}{l}\text { Incidence of diabetic } \\
\text { ketoacidosis or } \\
\text { hypoglycaemia }\end{array}$ \\
\hline & 30-day mortality & $\begin{array}{l}\text { incidence and duration of } \\
\text { use of intravenous insulin } \\
\text { infusion therapy }\end{array}$ \\
\hline & $\begin{array}{l}\text { Length of stay in } \\
\text { hospital }\end{array}$ & $\begin{array}{l}\text { Incidence of diabetic } \\
\text { ketoacidosis or } \\
\text { hypoglycaemia }\end{array}$ \\
\hline & $\begin{array}{l}\text { Length of stay in } \\
\text { ICU (if applicable) }\end{array}$ & $\begin{array}{l}\text { Change in diabetic } \\
\text { management at } 30 \text { days }\end{array}$ \\
\hline & $\begin{array}{l}\text { Incidence of } \\
\text { specific major } \\
\text { adverse events }\end{array}$ & \\
\hline
\end{tabular}

ICU, Intensive Care Unit.

- Length of stay in Intensive Care Unit (ICU) if applicable.

- Incidence of specific major adverse events as listed in European Perioperative Clinical Outcome definitions manuscript. ${ }^{17}$ These and other outcomes are shown in table 1.

\section{Data sources}

The following data will be extracted from clinical charts: age, gender, weight, height, variables for CCI and variables for Surgical Outcome Risk Tool (SORT) calculation (SORT Score).

American Society Anaesthesiolog (ASA) classification, relevant medical history, preoperative diabetes medication (substance classes only), type of anaesthesia, date, type and location of surgery, procedure duration, date of ICU admission and date of discharge from ICU.

A continuous glucose/insulin infusion will be regarded as planned, any insulin boluses on top of this infusion will be deemed rescue (or 'additional').

Bias-In every centre, all patients with diabetes undergoing surgery, except where there is only conscious sedation, with or topical anaesthesia to the eye or both combined, are eligible. Centres are invited to enrol their target number of patients (depending on number of investigators in their team) from date of registration of their centre with ESA for up to 18 months. Once they start to enrol patients, centres are asked to do so consecutively, that is, to take all eligible patients with diabetes one after another. No other exclusion criteria apply, even emergency surgery patients are eligible. Therefore, we do not believe that significant risk of bias exists. 


\section{Study procedures}

\section{Recruitment and screening}

At screening day ('day -90 ' to 'day of surgery', that is, within 3 months of planned day of surgery), patients may be screened and invited to participate. Patients with diabetes listed for both elective and emergency surgery are eligible. They will be offered a PIL and the investigator will withdraw to allow the patient to consider it by alone. The team member will obtain signed written consent if the patient agrees to proceed. While for elective patients, consent may be obtained in a preoperative clinic up to 90 days prior, for emergency surgery diabetic patients' consent may be requested on the ward, immediately prior to coming to theatre on the day of surgery. This is justified because there is even less knowledge currently about the management and outcomes of patients with diabetes undergoing emergency surgery, who are acknowledged to be a particularly high-risk group, compared with patients with diabetes undergoing elective surgery. Therefore, including a cohort of these patients is particularly important to evaluate risk factors for adverse outcomes which may be mitigated. There is also anecdotal evidence that practice of managing these patients varies widely between nations and individual centres. The SORT will be used to indicate surgical risk. ${ }^{17}$

If patients remain in hospital on the day after surgery, QoR-15 quality of recovery score will be documented. Patient data on insulin use, glucose levels and any complications observed will also be recorded on the day of discharge, provided a patient is discharged within 30 days of his/her surgery. At day 30 after surgery, data will be collected by telephone if the patient has been discharged. If still in hospital, patient data will be collected on the ward on day 30 , see figure 1 .

\section{Data collection}

At the end of the study period, each centre will provide an 'end of study reporting form' to report the number of patients meeting the inclusion criteria during the study period and the total number of screening failure patients. Furthermore, each centre will provide a screening failure tracking form giving the reasons for screening failures at the end of the study period. Using this form, it will be possible to analyse what are the reasons for exclusion from study (eg, subject refused to sign informed consent, subject is already participating in other clinical trial, subject language, cognitive difficulties, etc). Data will be collected at each centre, anonymised and entered into a bespoke electronic case-report form (eCRF). Completed forms will be submitted to the sponsor at the ESA Clinical Trials Network (ESA CTN) in Brussels, Belgium.

\section{Statistical analysis plan Primary outcome}

The primary outcome of the study will be the descriptive epidemiology of the perioperative management and postoperative morbidity of patients with diabetes across different countries in Europe. Morbidity and mortality will be assessed using DAH-30 as the primary outcome.

\section{Secondary outcomes}

Secondary outcomes will be morbidity as assessed by the CCI Score, based on Clavien-Dindo Scale and additional hypotheses of interest as listed in table 2.

\section{Sample size estimation}

Up to 5\% of the population of Europe is thought to have diabetes. About 30 million surgeries are performed in Europe per annum, therefore perhaps 1.5 million diabetics have surgery in Europe each year. It is proposed to evaluate a pragmatic sample of 5000 European patients with diabetes across at least 50 centres in a minimum of 10 nations. It is expected that this should be sufficient for the main epidemiological aspects of this study. It is envisaged that this target number will be enrolled over a 2-year period from initial roll-out, with up to a further 12 months needed for final data acquisition, data cleaning and analysis. A sample size of 5000 should be sufficient to avoid overfitting and variance inflation for $50-70$ factors and interactions based on the conventional square root or 100 values per variable, respectively. In addition, a sample size of 5000 will have at least $90 \%$ power to find a standardised difference of 0.15 as significant at $p<0.05$ (Bonferroni corrected at $\mathrm{p}<0.0007$ ) for up to 70 independent hypotheses and in comparing subsets of interest.

\section{Primary statistical analysis}

Descriptive statistics such as mean (SD), median (IQR) and frequencies (\%) will be presented as appropriate. Gaussian distributions will be assessed using frequency histograms, normality plots and the Shapiro-Wilk statistic. The precision of the estimates will be reported as 95\% CIs to show the prevalence and incidence rates of diabetic phenotypes and major adverse events and complications.

Continuous data will be analysed using Student's t-test, Mann-Whitney U test, one-way analysis of variance and Kruskal-Wallis $\mathrm{H}$ statistics. Categorical data will be analysed using $\chi^{2}$ independence and expanded Fisher exact statistics. Multiple hypothesis or comparison testing will be addressed using Tukey-Kramer and Bonferroni corrections and overall statistical significance will be defined at $\mathrm{p}<0.05$ (two sided).

Repeated measurements in patients will be analysed using generalised linear mixed models (GLMM) with maximum likelihood estimation using appropriate link functions: Gaussian, Poisson, negative binomial and logit. Robust multivariable linear, logistic, proportional hazards and quantile regression models will be constructed to identify significant independent risk factors for adverse outcomes. Variables with $\mathrm{p}<0.15$ on bivariate analysis, or that are clinically relevant, will be entered. Multicollinearity will be assessed using variance inflation factors. Hierarchical nesting of patients in hospitals and countries will be entered as random effects in multilevel mixed effects GLMM. 


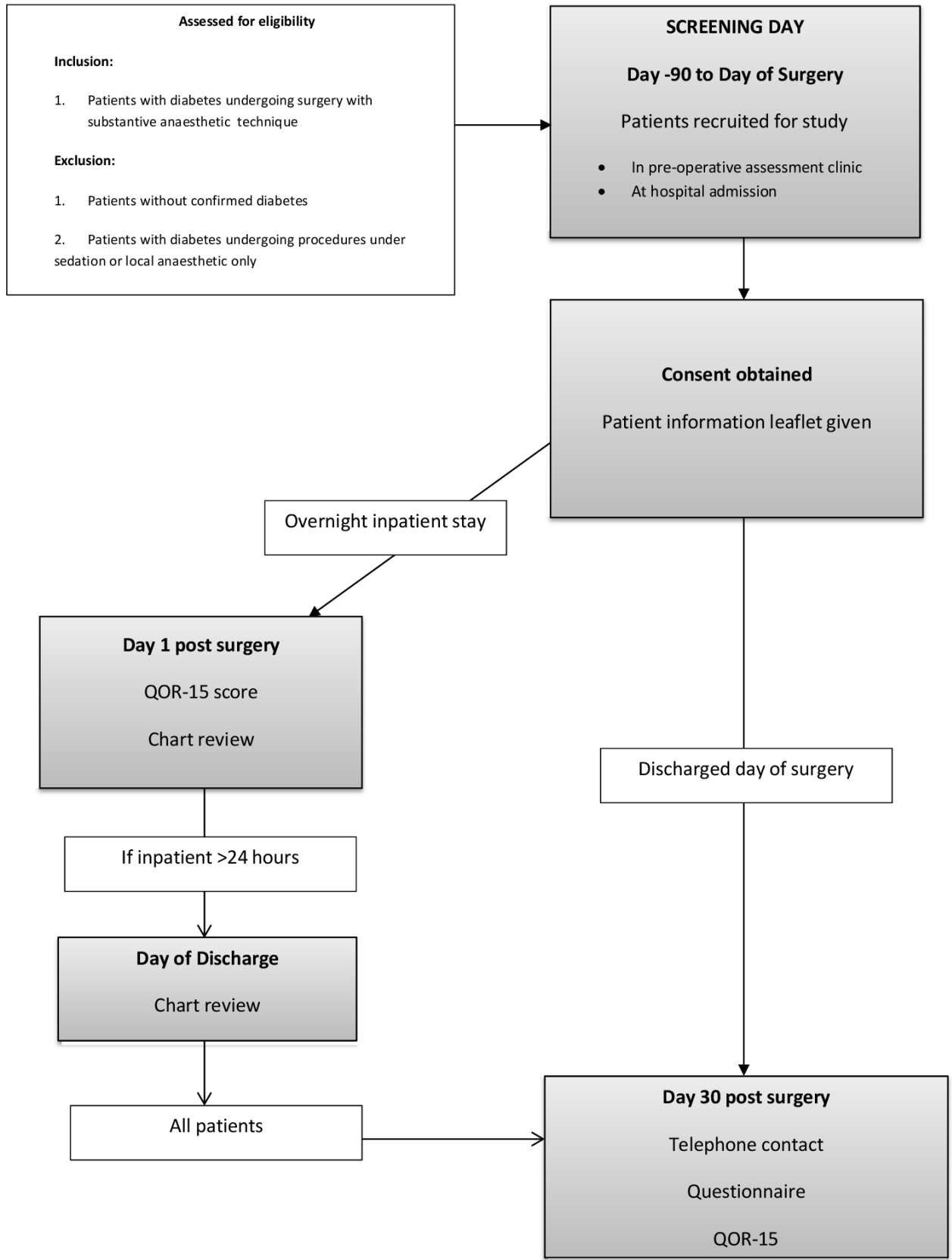

Figure 1 Study work flow. QoR-15, Quality of Recovery Scale.

\section{Secondary statistical analysis}

Exploratory post hoc analyses may be performed to gain further information about the cohort and to assess clinical outcomes with respect to participating countries and hospitals. Any post hoc analyses will be identified as such in any reports. Participating institutions can request data extraction for further analysis and quality improvement, subject to approval of the SC. As the primary purpose of this project is epidemiological, missing data will not be replaced or imputed.

\section{Software}

Data will be analysed using Stata V.16.1, StataCorp, College Station, Texas, and Number Cruncher Statistical Systems 2020, NCSS, Kaysville, Utah.
The sponsor and the SC have the right to veto the nesting of a study into MOPED. The publication of any study nested within MOPED will occur after publication of the main results of MOPED (main objectives 1 and 2 ). For transparency, the original paper should be referenced to in all articles of nested analyses. Authorship rules for potential publications derived from such nested cohort studies are to be submitted to the sponsor and SC together with the study proposal.

Requests for data sharing for individual-level metaanalyses are to be addressed to the sponsor and SC.

The sponsor of the study (ESA CTN) can use anonymised pooled data for internal analyses and educational purposes. 
Table 2 Secondary outcomes and hypotheses of interest

\begin{tabular}{ll}
\hline Hypothesis & Variables \\
\hline $\begin{array}{l}\text { There are major differences in perioperative management of patients } \\
\text { with diabetes in different nations in Europe }\end{array}$ & $\begin{array}{l}\text { Insulin dose } \\
\text { Methods of insulin admin } \\
\text { Oral hypoglycaemic use }\end{array}$ \\
$\begin{array}{ll}\text { There are major differences in postoperative morbidity and outcomes } \\
\text { among patients with diabetes in different nations in Europe }\end{array}$ & $\begin{array}{l}\mathrm{DAH}-30 \\
\mathrm{CCl}\end{array}$ \\
$\begin{array}{l}\text { Outcomes among patients with different strata of glycaemic control, } \\
\text { that is, }\end{array}$ & $\begin{array}{l}\text { Preoperative HbA1c and glucose } \\
\text { DAH-30 }\end{array}$ \\
$\begin{array}{ll}\mathrm{HbA} 1 \mathrm{c}<53 \mathrm{mmol} \cdot \mathrm{mol}, \\
\mathrm{Hb} 1 \mathrm{c} 53-69 \mathrm{mmol} \cdot \mathrm{mol} \text { and }\end{array}$ & \\
$\mathrm{HbA1c}>69 \mathrm{mmol} \cdot \mathrm{mol}$ will be different & \\
$\begin{array}{l}\text { Diabetic patient outcomes differ depending on anaesthetic technique: } \\
\text { Volatile versus total intravenous anaesthesia }\end{array}$ & $\mathrm{DAH}-30$ \\
Regional versus general anaesthesia (GA) & $\mathrm{CCl}$ \\
\hline
\end{tabular}

Combined GA and regional anaesthesia versus patients receiving GA alone

Patients with diabetes receiving liberal fluids perioperatively have better DAH-30, CCI crystalloid and colloid totals up to PACU outcomes than patients receiving restrictive fluids, compared with their body weight

Type 2 DM patients have worse outcomes than type 1

DAH-30, CCI

Patients where a consultant/senior surgeon and senior anaesthesiologist is present have better outcomes than when not present

Patients with diabetes of longer duration experience more hypotension duration/episodes due to autonomic neuropathy and have worse outcomes than patients with diabetes with shorter duration

Personnel tracking All outcomes

NSAID use perioperatively worsens outcomes especially AKI

Intraoperative and PACU hypotension and use vasopressors and outcomes

Duration of DM

Risk factors for higher morbidity in patients with diabetes undergoing
surgery

DAH-30, CCI

AKI

All factors

All outcomes

Multivariable analysis

\begin{tabular}{|c|c|}
\hline $\begin{array}{l}\text { Patients with preoperative GLP-1 use have better perioperative glucose } \\
\text { control (and outcome) as compared with other oral hypoglycaemics }\end{array}$ & $\begin{array}{l}\text { Preoperative medication use } \mathrm{DAH}-30 \\
\mathrm{CCl}\end{array}$ \\
\hline $\begin{array}{l}\text { There is no association between metformin use and perioperative lactic } \\
\text { acidosis }\end{array}$ & $\begin{array}{l}\text { Preoperative medication use } \\
\text { Incidence of DKA } \\
\text { DAH-30 } \\
\text { CCI }\end{array}$ \\
\hline $\begin{array}{l}\text { Patients with known preoperative susceptibility for hypoglycaemia/DKA } \\
\text { are more prone for perioperative hypoglycaemia/DKA }\end{array}$ & $\begin{array}{l}\text { Preoperative hypoglycaemia/DKA } \\
\text { Perioperative hypoglycaemia/DKA }\end{array}$ \\
\hline Surgery in DM will lead to dysglycaemia up to 30 days & DM medication at 30 days \\
\hline
\end{tabular}

AKI, Acute Kidney Injury; CCI, Comprehensive Complications Index; DAH-30, days at home at 30 days; DKA, Diabetic Ketoacidosis; DM, Diabetes; GLP, Glycogen Like Peptide; HbA1c, haemoglobin A1c; NSAID, Non-Steroidal Anti-Inflammatory Drugs; PACU, Post-Anaesthesia Care Unit.

\section{GDPR, data and quality management}

Quality control measures will be applied to each stage of data handling to ensure that all data are reliable and have been processed correctly. This will include written Standard Operating Procedure (SOP) (in English for all countries) for data collection and entry, automated consistency checks and training of national co-ordinating investigator and local Principal Investigator (PI). It will be the responsibility of the national co-ordinating investigator, with support by the study co-ordinating office, to train local PIs. Local centre co-ordinators will ensure that the data in the eCRF are carefully entered and verified regularly. It will be the responsibility of local co-ordinators to conduct periodic and random checks to ensure data quality in that centre. The ESA as sponsor is responsible for securing agreement from all involved parties to ensure direct access to all trial related sites and source documents for the purpose of monitoring and auditing. No fee or financial compensation is given to any coinvestigator or participating institution for patient recruitment.

Data handling-Data will be entered into a secure online database protected by personalised and confidential 
usernames and passwords, which document the time and the individual entering the data. The language of the online database, eCRF and the relative SOPs is English and will not be translated into different languages. Data will be collected directly from source documents into the encoded paper CRF and secondarily entered into the eCRF. A copy of the original source documents will be stored within a locked cabinet/office accessible to authorised personnel only in accordance with local and national regulations. All study documents will be archived as required by local legislation. Sponsor and centres will maintain and update their trial master files according to the recommendation of the ICH-GCP Guidelines E6(R2).

Confidentiality and data protection-To safeguard patients' confidentiality, a patient identification code will be assigned to encode data. The confidential log linking patient identification codes and identifiable patient data will be stored separately in a locked cabinet accessible to authorised personnel only and corresponding electronic files will be protected by personalised and confidential usernames and passwords. eCRF are identified through the patient identification code and will not include any names, initials, date of birth or local hospital patient numbers. Therefore, no patient identifiable data will be directly accessible from the eCRF. Open direct access to all relevant trial information as well as source data/documents will be permitted for purposes of monitoring, audits or inspections by the sponsor, national co-ordinators, IRB or regulatory authorities. All handling of personal data will comply with the GCP Guidelines and follow strictly the legal and national requirements for data protection.

\section{Patient and public involvement}

To maximise the benefit of this study to patients, we prioritised using a patient-centric, holistic primary outcome: DAH-30. Previous Delphi process driven studies have shown this to be a sensitive index of postoperative complications and their impact on patients' lives. Ireland's diabetes patient advocacy association, Diabetes Ireland, kindly reviewed the draft protocol and offered comment and suggestion which influenced the final draft.

\section{Publication and dissemination of results}

The main results of MOPED and its substudies will be published in peer-reviewed international medical journals and presented at Euroanaesthesia and at international and national meetings. As recommended by the International Committee of Medical Journal Editors (http:// www.icmje.org/recommendations/browse/roles-andresponsibilities/defining-the-role-of-authors-and-contributors.html; accessed 30 August 2016), authorship will be considered based on contributions to recruitment of patients, data acquisition and cleaning, analysis and interpretation of data, manuscript writing and submission of national/local grants. Authors are required to give final approval of the version to be published and agree to be accountable for all aspects of the work in ensuring that questions related to the accuracy or integrity of any part of the work are appropriately investigated and resolved. The SC will also be the writing committee (WC).

All papers derived from the MOPED database will be published under the acronym 'The MOPED Investigators'. All authors will be specifically named, in order to give every investigator the same credit and the same responsibilities for successfully performing this study. All authors will be mentioned with their name and affiliation in the collaborators list which will be published to the manuscript. The members of the SC will be specifically identified as required by most journals. Collaborators names will be listed in PubMed.

It is the responsibility of the local co-ordinators to determine who is to be considered as investigator. The local PI will be asked to submit names of staff actively involved from their institution in the end of study reporting form. If the number of recruited patients from a centre is too low to justify sufficient active involvement, the SC may decide on the legitimacy of collaboratorship based on other contributions. The final decision will be left to the SC in consultation with the ESA. The number of investigators allowed from each centre will be determined by the number of patients enrolled by that centre. No more than $25 \%$ of a centre's enrolled patients should be day cases (ambulatory anaesthesia).

Presentation at international meetings will be restricted to the members of the SC or their delegates. National co-ordinators will qualify for presentation at national meetings after approval by the SC and the sponsor. ESA Clinical Trial Network will be acknowledged in all publications and presentations.

After publication of the pooled results, centres will be allowed to use their own anonymised data for local presentation and publication. Duplicate data publication is not permitted.

\section{Author affiliations}

${ }^{1}$ Dept. Anaesthesiology, Mater University Hospital, University College Dublin, Dublin, Ireland

${ }^{2}$ Mater University Hospital, University College Dublin, Dublin, Ireland

${ }^{3}$ Outcomes Research, Cleveland Clinic, Cleveland, OH, USA

${ }^{4}$ University of Bonn, Bonn, Germany

${ }^{5}$ Wythenshawe Hospital Acute Intensive Care Unit, Manchester University NHS Foundation Trust, Manchester, UK

${ }^{6}$ Department of Anesthesiology, Amsterdam University Medical Center, Duivendrecht, Noord-Holland, The Netherlands

${ }^{7}$ Universität Münster, Münster, UK

Contributors DJB, MCoburn, MColumb, JH, MVH and AZ devised the project, contributed to the design of the study and developed the protocols for data collection and analysis. DJB, RN, MCoburn and MColumb were involved in the writing of the manuscript. All authors gave final approval to the publishing of this work and agree to be accountable for the integrity and veracity of this protocol and the data collected and analysed thereafter.

Funding European Society of Anaesthesia as study sponsor is providing administrative support for data collating.

Competing interests None declared.

Patient and public involvement Patients and/or the public were not involved in the design, or conduct, or reporting, or dissemination plans of this research.

Patient consent for publication Not required. 
Provenance and peer review Not commissioned; externally peer reviewed.

Open access This is an open access article distributed in accordance with the Creative Commons Attribution Non Commercial (CC BY-NC 4.0) license, which permits others to distribute, remix, adapt, build upon this work non-commercially, and license their derivative works on different terms, provided the original work is properly cited, appropriate credit is given, any changes made indicated, and the use is non-commercial. See: http://creativecommons.org/licenses/by-nc/4.0/.

\section{ORCID iDs}

Donal J Buggy http://orcid.org/0000-0003-3922-4397

Rachel Nolan http://orcid.org/0000-0001-9644-8958

Alexander Zarbock http://orcid.org/0000-0002-2124-1714

\section{REFERENCES}

1 Cho NH, Kirigia J, Claude J, International diabetes federation. IDF diabetes atlas. $8^{\text {th }}$ ed, 2019. http://www.diabetesatlas.org/resources/ 2017-atlas.html

2 Frisch A, Chandra P, Smiley D, et al. Prevalence and clinical outcome of hyperglycemia in the perioperative period in noncardiac surgery. Diabetes Care 2010;33:1783-8.

3 Hulst $\mathrm{AH}$, Hermanides J, Hollmann MW, et al. Lack of consensus on the peri-operative management of patients with diabetes mellitus. Eur J Anaesthesiol 2019;36:168-9.

4 Plummer MP, Bellomo R, Cousins CE, et al. Dysglycaemia in the critically ill and the interaction of chronic and acute glycaemia with mortality. Intensive Care Med 2014;40:973-80.

5 Umpierrez GE, Smiley D, Jacobs S, et al. Randomized study of basal-bolus insulin therapy in the inpatient management of patients with type 2 diabetes undergoing general surgery (rabbit 2 surgery). Diabetes Care 2011;34:256-61.

6 Martin ET, Kaye KS, Knott C, et al. Diabetes and risk of surgical site infection: a systematic review and meta-analysis. Infect Control Hosp Epidemiol 2016;37:88-99.
7 Vascular Events in Noncardiac Surgery Patients Cohort Evaluation (VISION) Study Investigators, Spence J, LeManach Y, et al. Association between complications and death within 30 days after noncardiac surgery. CMAJ 2019;191:E830-7.

8 Kuzulugil D, Papeix G, Luu J, et al. Recent advances in diabetes treatments and their perioperative implications. Curr Opin Anaesthesiol 2019;32:398-404.

9 National Confidential enquiry into patient outcome and death. highs and Lows, London, 2018. Available: https://www.ncepod.org.uk/ 2018pd/Highs\%and\%20Lows_Summary\%20Report.pdf [Accessed 22 Jan 2020].

10 Garg R, Schuman B, Bader A, et al. Effect of preoperative diabetes management on glycemic control and clinical outcomes after elective surgery. Ann Surg 2018;267:858-62.

11 Gatti G, Perrotti A, Reichart D, et al. Glycated hemoglobin and risk of sternal wound infection after isolated coronary surgery. Circ $J$ 2016;81:36-43

12 Myles PS, Shulman MA, Heritier S, et al. Validation of days at home as an outcome measure after surgery: a prospective cohort study in Australia. BMJ Open 2017;7:E015828.

13 Bell M, Eriksson LI, Svensson T, et al. Days at home after surgery: an integrated and efficient outcome measure for clinical trials and quality assurance. EClinicalMedicine 2019;11:18-26.

14 Slankamenac K, Nederlof N, Pessaux P, et al. The comprehensive complication index: a novel and more sensitive endpoint for assessing outcome and reducing sample size in randomized controlled trials. Ann Surg 2014;260:757-62. discussion 762-3.

15 Clavien P-A, Vetter D, Staiger RD, et al. The Comprehensive Complication Index (CCI $(\mathbb{)})$ : Added Value and Clinical Perspectives 3 Years "Down the Line". Ann Surg 2017;265:1045-50.

16 Stark PA, Myles PS, Burke JA. Development and psychometric evaluation of a postoperative quality of recovery score: the QoR-15. Anesthesiology 2013;118:1332-40.

17 Protopapa KL, Simpson JC, Smith NCE, et al. Development and validation of the surgical outcome risk tool (SORT). Br J Surg 2014;101:1774-83. 